\title{
A new inverse Weibull distribution: properties, classical and Bayesian estimation with
}

\section{applications}

\author{
Ahmed Z. Afify ${ }^{1}$, Ahmed I. Shawky ${ }^{2}$, Mazen Nassar ${ }^{2,3, *}$ \\ ${ }^{1}$ Dept. of Statistics, Mathematics and Insurance, Benha University, Benha 13511, Egypt \\ ${ }^{2}$ Dept. of Statistics, Faculty of Science, King Abdulaziz University, Jeddah, Saudi Arabia \\ ${ }^{3}$ Dept. of Statistics, Faculty of Commerce, Zagazig University, Egypt \\ *Corresponding author: mezo10011@gmail.com
}

\begin{abstract}
This article proposes a new extension of the inverse Weibull distribution called, logarithmic transformed inverse Weibull distribution which can provide better fits than some of its well-known extensions. The proposed distribution contains inverse Weibull, inverse Rayleigh, inverse exponential, logarithmic transformed inverse Rayleigh and logarithmic transformed inverse exponential distributions as special sub-models. Our main focus is to derive some of its mathematical properties along with the estimation of its unknown parameters using frequentist and Bayesian estimation methods. We compare the performances of the proposed estimators using extensive numerical simulations for both small and large samples. The importance and potentiality of this distribution is analyzed via two real data sets.
\end{abstract}

Keywords: Bayesian estimation; inverse Weibull distribution; moments; quantile function; maximum product of spacings estimation.

\section{Introduction}

The inverse Weibull distribution (IW) takes some different names like Fréchet distribution, see Johnson et al., 1995. Okasha et al., 2017, introduced Marshall-Olkin-IW among others. In this article, we propose a new three-parameter extension of the IW distribution, referred to as logarithmic transformed-IW (LTIW) distribution that is constructed using the logarithmic transformed-G (LT-G) family introduced by Nassar et al., 2018. The LTIW distribution is motivated by the following desirable properties. (1) The LTIW is a comprehensive model which contains some well-known sub-models. (2) It is shown in Section 2 that the LTIW distribution can be viewed as a mixture of the IW distribution. (3) The LTIW distribution can provide increasing, decreasing and unimodal hazard rate which makes it superior to some other distributions. (4) The LTIW can be considered as a suitable model for modeling skewed data.

The cumulative distribution function (CDF) of the LT-G class is given by

$$
F_{L T}(x ; \delta)=1-\frac{\log \left[1+\delta-\delta^{G(x)}\right]}{\log (\delta)}, \quad \delta \neq 1
$$

and its corresponding PDF has the form

$$
f_{L T}(x ; \delta)=\frac{g(x) \delta^{G(x)}}{1+\delta-\delta^{G(x)}}, \quad \delta \neq 1,
$$

where $G(x)$ and $g(x)$ are the $\mathrm{CDF}$ and probability density function (PDF) of a baseline random variable $\mathrm{X}$, respectively.

The paper is organized as follows. We present the proposed LTIW distribution and provide its special sub-models in Section 2. In Sections 3, we derive some properties of the LTIW distribution. Section 4 describes eight classical methods of estimation along with the 
Bayes approach. In Section 5, we provide simulation results to compare the performance of the estimation methods for the LTIW model. In Section 6, the importance of the LTIW distribution is discussed using two real-life data sets. Finally, Section 7 provides some concluding remarks.

\section{The proposed distribution}

By inserting the CDF of the IW model, $G(x ; \alpha, \lambda)=\exp \left(-\lambda x^{-\alpha}\right), x>0, \alpha, \lambda>0$, then the CDF of LTIW distribution reduces to

$$
\begin{aligned}
F(x ; \delta, \alpha, \lambda)= & 1-\frac{\log \left[1+\delta-\delta^{\exp \left(-\lambda x^{-\alpha}\right)}\right]}{\log (\delta)}, \\
& x>0, \alpha, \lambda, \delta>0 .
\end{aligned}
$$

(3) Fig. 1. Plots of the LTIW density for $\lambda=1$ and

some values of $\alpha$ and $\delta$.

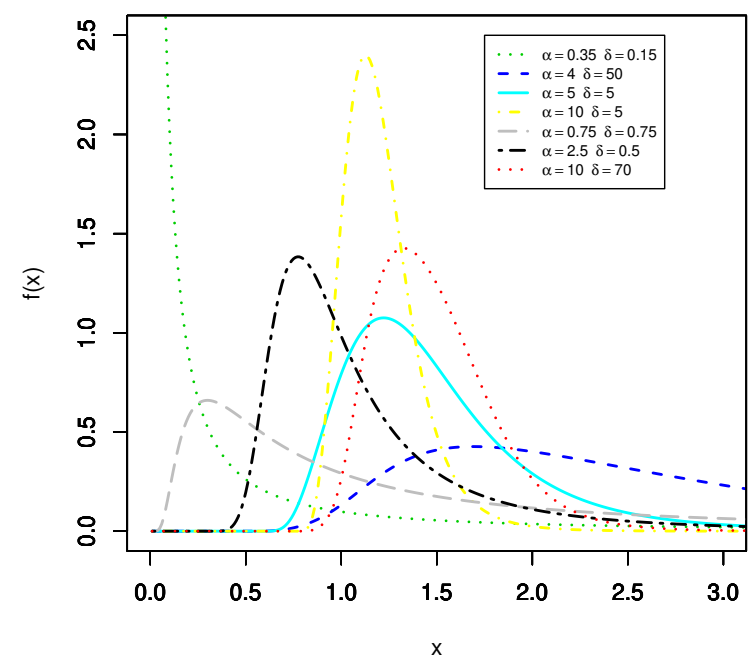

The associated PDF of (3) has the form

$$
\begin{aligned}
f(x ; \delta, \alpha, \lambda) & =\frac{\alpha \lambda x^{-\alpha-1} \exp \left(-\lambda x^{-\alpha}\right)}{1+\delta-\delta^{\exp \left(-\lambda x^{-\alpha}\right)}} \\
& \times \delta^{\exp \left(-\lambda x^{-\alpha}\right)}
\end{aligned}
$$

Its hazard rate function (HRF) of $X$ reduces to

$$
\begin{aligned}
h(x ; \delta, \alpha, \lambda) & =\frac{\log (\delta) \alpha \lambda x^{-\alpha-1} \exp \left(-\lambda x^{-\alpha}\right)}{\left[1+\delta-\delta^{\exp \left(-\lambda x^{-\alpha}\right)}\right]} \\
& \times \frac{\delta^{\exp \left(-\lambda x^{-\alpha}\right)}}{\log \left[1+\delta-\delta^{\exp \left(-\lambda x^{\alpha}\right)}\right]} .
\end{aligned}
$$

Hereafter, the random variable $(r v) X$ having the PDF (4) is referred to as $X \sim \operatorname{LTIW}(\delta, \alpha, \lambda)$. Figures 1 and 2 show some shapes for the PDF and HRF plots of the LTIW model, for different parametric values.

\section{Properties of the LTIW model}

\subsection{Quantile function}

The QF of the LTIW distribution follows, by inverting its CDF in (3), as

$$
\begin{aligned}
Q(p)= & \left\{\frac{-1}{\lambda} \log \left[\frac{\log \left(1+\delta-\delta^{1-p}\right)}{\log (\delta)}\right]\right\}^{-1 / \alpha}, \\
& 0<p<1
\end{aligned}
$$

Hence, simulating the LTIW $r v$ is simply straightforward. The $r v X=Q(P)$ has PDF

\subsection{Linear representation}

Here, we obtain a useful linear representation for the LTIW density based on the two power series defined by

$$
\begin{aligned}
(1-z)^{-1}= & \sum_{l=0}^{\infty} z^{l},|z|<1 \\
& \delta^{z}=\sum_{j=0}^{\infty} \frac{[\log (\delta)]^{j}}{j !} z^{j}
\end{aligned}
$$

Applying these two power series, the LTIW PDF reduces to

$$
\begin{aligned}
f(x ; \alpha, \lambda, \delta) & =\alpha \lambda x^{-\alpha-1} \sum_{l, j=0}^{\infty} \frac{[\log (\delta)]^{j}(l+1)^{j}}{j !(1+\delta)^{l+1}} \\
& \times \exp \left(-(j+1) \lambda x^{-\alpha}\right) .
\end{aligned}
$$

Simply, the PDF of the LTIW distribution takes the form

$$
f(x ; \alpha, \lambda, \delta)=\sum_{j=0}^{\infty} \eta_{j} g_{(j+1) \lambda, \alpha}(x),
$$




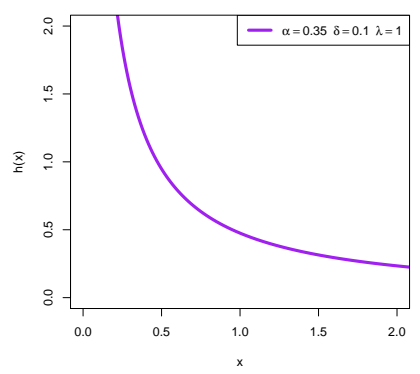

form

$$
\mu_{n}^{\prime}=\sum_{j=0}^{\infty} \eta_{j}[(j+1) \lambda]^{\frac{n}{\alpha}} \Gamma\left(1-\frac{n}{\alpha}\right) .
$$

The $n$th incomplete moment of LTIW distribution follows, from Equation (5), as

$$
\begin{aligned}
\varphi_{n}(t) & =\sum_{j=0}^{\infty} \eta_{j}[(j+1) \lambda]^{\frac{n}{\alpha}} \\
& \times \gamma\left(1-\frac{n}{\alpha},(j+1) \lambda t^{-\alpha}\right) .
\end{aligned}
$$

The first incomplete moment, denoted by $\varphi_{1}(t)$, follows, by setting $n=1$ in the above equation, as

$$
\begin{aligned}
\varphi_{1}(t) & =\sum_{j=0}^{\infty} \eta_{j}[(j+1) \lambda]^{\frac{1}{\alpha}} \\
& \times \gamma\left(1-\frac{1}{\alpha},(j+1) \lambda t^{-\alpha}\right) .
\end{aligned}
$$

3.4 Mean residual life and mean inactivity time

The MRL for the LTIW distribution reduces to

$$
m_{X}(t)=\left[1-\varphi_{1}(t)\right] / S(t)-t,
$$

Fig. 2. HRF plots of the LTIW distribution for different parametric values of $\alpha, \delta$ and $\lambda$.

where $g_{(j+1) \lambda, \alpha}(x)$ is the IW density with two parameters $(j+1) \lambda$ and $\alpha$, and $\eta_{j}$ has the form

$$
\eta_{j}=\frac{[\log (\delta)]^{j}}{(j+1) !} \sum_{l=0}^{\infty} \frac{(l+1)^{j}}{(1+\delta)^{l+1}} .
$$

\subsection{Moments}

Henceforth, let $Z \sim \operatorname{IW}(\alpha, \lambda)$ with PDF $f(z)=$ $\alpha \lambda z^{-\alpha-1} e^{-\lambda z^{-\alpha}}$, where $\alpha, \lambda>0$. Hence, the $n$th ordinary and incomplete moments of $Z$ take the following forms

$$
\begin{aligned}
& m u_{n, Z}^{\prime}=\lambda^{\frac{n}{\alpha}} \Gamma\left(1-\frac{n}{\alpha}\right), \\
& \varphi_{n, Z}(t)=\lambda^{\frac{n}{\alpha}} \gamma\left(1-\frac{n}{\alpha}, \lambda t^{-\alpha}\right),
\end{aligned}
$$

respectively. Using Equation (5), the $n$th ordinary moment of the LTIW model has the

where $\varphi_{1}(t)$ is the first incomplete moment of the LTIW distribution given by (6) and $S(t)=$ $1-F(t)$ refers to its survival function (SF). Then, the MRL of the LTIW distribution, follows by combining (6) and (7), as

$$
\begin{aligned}
m_{X}(t) & =\frac{1}{S(t)} \sum_{j=0}^{\infty} \eta_{j}[(j+1) \lambda]^{\frac{1}{\alpha}} \\
& \times \gamma\left(1-\frac{1}{\alpha},(j+1) \lambda t^{-\alpha}\right)-t .
\end{aligned}
$$

The MIT of the $r v X$ has the form

$$
M_{X}(t)=t-\left[\varphi_{1}(t) / F(t)\right]
$$

Hence, the MIT of the LTIW distribution follows, by inserting Equation (6) in (8), as

$$
\begin{aligned}
M_{X}(t) & =t-\frac{1}{F(t)} \sum_{j=0}^{\infty} \eta_{j}[(j+1) \lambda]^{\frac{1}{\alpha}} \\
& \times \gamma\left(1-\frac{1}{\alpha},(j+1) \lambda t^{-\alpha}\right) .
\end{aligned}
$$




\subsection{Generating function}

Afify et al., 2016, derived a simple formula for the MGF of the Fréchet (Fr) distribution with $\operatorname{CDF} G(x ; \alpha, \lambda)=\exp \left(-\left(\frac{\lambda}{x}\right)^{\alpha}\right), x>0, \alpha, \lambda>$ 0 , as follows

$$
M_{F r}(t ; \alpha, \lambda)={ }_{1} \Psi_{0}\left[\begin{array}{c}
\left(1,-\alpha^{-1}\right) \\
-
\end{array}\right] \lambda t .
$$

Hence, the MGF of the IW with CDF, $G(x ; \alpha, \lambda)=\exp \left(-\lambda x^{-\alpha}\right), x>0, \alpha, \lambda>0$, follows simply from (9) as

$$
M_{I W}(t ; \alpha, \lambda)={ }_{1} \Psi_{0}\left[\begin{array}{c}
\left(1,-\alpha^{-1}\right) \\
-
\end{array} ; \lambda^{\frac{1}{\alpha}} t\right] .
$$

Hence, the MGF of the LTIW model, $M_{X}(t)$, follows by combining the two Equations (5) and (10), as

$$
M_{X}(t)=\sum_{j=0}^{\infty} \eta_{j 1} \Psi_{0}\left[\begin{array}{c}
\left(1,-\alpha^{-1}\right) \\
-
\end{array}\right],
$$

where $V=[(j+1) \lambda]^{\frac{1}{\alpha}} t$.

\subsection{Order statistics}

The PDF and CDF of the $r$ th order statistic $X_{r: n}$, $f_{r: n}(x)$, take the forms

$$
\begin{aligned}
f_{r: n}(x) & =\frac{n !}{(r-1) !(n-r) !}[F(x)]^{r-1} \\
& \times[1-F(x)]^{n-r} f(x), r=1,2, . ., n
\end{aligned}
$$

and

$$
F_{r: n}(x)=\frac{n !}{(r-1) !(n-r) !} \sum_{i=0}^{n-r} \frac{(-1)^{r}}{(r+i)}\left(\begin{array}{c}
n-r \\
i
\end{array}\right) \quad-\sum_{i=1}^{n} \log \left[1+\delta-\delta^{\exp \left(-\lambda x_{i}^{-\alpha}\right)}\right]_{1.2)}
$$$$
[F(x)]^{r+i}, r=1,2, . ., n \text {. }
$$

The PDF of $X_{r: n}$ for a LTIW distribution is

$$
\begin{aligned}
f_{r: n}(x) & =\frac{n ! \alpha \lambda}{(r-1) !(n-r) !} \sum_{i=0}^{r-1}(-1)^{i}\left(\begin{array}{c}
r-1 \\
i
\end{array}\right) \\
& \times\left\{\frac{\log [\Psi(x ; \delta, \alpha, \lambda)]}{\log (\delta)}\right\}^{n-r+i} \\
& \times \frac{x^{-\alpha-1} \exp \left(-\lambda x^{-\alpha}\right) \delta^{\exp \left(-\lambda x^{-\alpha}\right)}}{\Psi(x ; \delta, \alpha, \lambda)}
\end{aligned}
$$

where $\Psi(x ; \delta, \alpha, \lambda)=1+\delta-\delta^{\exp \left(-\lambda x^{-\alpha}\right)}$. The associated CDF of $X_{r: n}$ for a LTIW distribution has the form

$$
\begin{aligned}
F_{r: n}(x) & =\frac{n !}{(r-1) !(n-r) !} \sum_{i=0}^{n-r} \frac{(-1)^{r}}{(r+i)} \\
& \left(\begin{array}{c}
n-r \\
i
\end{array}\right)\left\{1-\frac{\log [\Psi(x ; \delta, \alpha, \lambda)]}{\log (\delta)}\right\}^{r+i} .
\end{aligned}
$$

\section{Methods of estimation}

This section is devoted to describe nine methods of estimation for estimating the LTIW distribution parameters.

\subsection{Method of maximum likelihood}

Let $x_{1}, \ldots, x_{n}$ be a random sample of size $n$ from the LTIW model, then the likelihood function of the parameter vector $\boldsymbol{\theta}=(\delta, \alpha, \lambda)^{\top}$ is given by

$$
\begin{aligned}
L(\boldsymbol{\theta}) & =\lambda^{n} \alpha^{n} \exp \left(-\lambda \sum_{i=1}^{n} x_{i}^{-\alpha}\right) \\
& \times \prod_{i=1}^{n} \frac{x_{i}^{-\alpha-1} \delta^{\exp \left(-\lambda x_{i}^{-\alpha}\right)}}{1+\delta-\delta^{\exp \left(-\lambda x_{i}^{-\alpha}\right)}}
\end{aligned}
$$

The log-likelihood function of (11) can be written as follows

$$
\begin{aligned}
\log L(\boldsymbol{\theta}) & =n \log (\alpha \lambda)-(\alpha+1) \sum_{i=1}^{n} \log \left(x_{i}\right) \\
& -\lambda \sum_{i=1}^{n} x_{i}^{-\alpha}+\log (\delta) \sum_{i=1}^{n} e^{\left(-\lambda x_{i}^{-\alpha}\right)}
\end{aligned}
$$

The maximum likelihood estimators (MLEs) of $\delta, \alpha$ and $\lambda$, can be obtained numerically by maximizing (12) with respect to $\delta, \alpha$ and $\lambda$.

\subsection{Method of percentiles}

If $\rho_{i}$ denotes an estimate of $F\left(x_{i: n} \mid \boldsymbol{\theta}\right)$, where $x_{1: n}, \ldots, x_{n: n}$ denote the order statistics of a random sample of size $n$ from the LTIW distribution, then the percentile estimators of the unknown parameters can be obtained by 
minimizing, with respect to $\delta, \alpha$ and $\lambda$, the following function

$$
\begin{aligned}
P(\boldsymbol{\theta}) & =\sum_{i=1}^{n}\left\{x_{i: n}-\lambda^{\alpha}\right. \\
& \left.\times\left[-\log \left(\frac{\log \left(y_{i}\right)}{\log (\delta)}\right)\right]^{-1 / \alpha}\right\}^{2}
\end{aligned}
$$

where $\rho_{i}=\frac{i}{n+1}$ is an unbiased estimator of $F\left(x_{i: n} \mid \boldsymbol{\theta}\right)$ and $y_{i}=1+\delta-\delta^{1-\rho_{i}}$. Instead of minimizing (13), we can obtain percentile estimators by solving the following system of non-linear equations

$$
\begin{aligned}
0 & =\sum_{i=1}^{n}\left\{x_{i: n}-\lambda^{\alpha}\left[-\log \left(\frac{\log \left(y_{i}\right)}{\log (\delta)}\right)\right]^{\frac{-1}{\alpha}}\right\} \\
& \times \quad \eta_{k i}, k=1,2,3,
\end{aligned}
$$

where

$$
\begin{gathered}
\eta_{1 i}=\frac{\lambda^{\alpha}\left[\frac{\log \left(y_{i}\right)}{\delta \log (\delta)}-\frac{1+\frac{\rho_{i}-1}{\delta_{i}}}{y_{i}}\right]}{\alpha \log \left(y_{i}\right) \varpi_{i}^{1+1 / \alpha}}, \\
\eta_{2 i}=\frac{\lambda^{\alpha}\left[\log (\lambda)+\alpha^{-2} \log \left(\varpi_{i}\right)\right]}{\varpi_{i}^{1 / \alpha}}
\end{gathered}
$$

and

$$
\eta_{3 i}=\frac{\alpha \lambda^{\alpha-1}}{\varpi_{i}^{1 / \alpha}},
$$

where $\varpi_{i}=-\left[\log \left(\log \left(y_{i}\right)\right)-\log (\log (\delta))\right]$.

\subsection{Method of maximum product of spacings}

Let us define the uniform spacings of a random sample from the LTIW distribution as:

$$
\begin{aligned}
D_{i}(\boldsymbol{\theta}) & =F\left(x_{i: n} \mid \boldsymbol{\theta}\right)-F\left(x_{i-1: n} \mid \boldsymbol{\theta}\right) \\
& =\frac{\log \left[1+\delta-\delta^{\left.\exp \left(-\lambda x_{i-1: n}^{-\alpha}\right)\right]}\right.}{\log (\delta)} \\
& -\frac{\log \left[1+\delta-\delta^{\left.\exp \left(-\lambda x_{i: n}^{-\alpha}\right)\right]}\right.}{\log (\delta)},
\end{aligned}
$$

where $F\left(x_{0: n} \mid \boldsymbol{\theta}\right)=0, F\left(x_{n+1: n} \mid \boldsymbol{\theta}\right)=$ 1 and $\sum_{i=1}^{n+1} D_{i}(\boldsymbol{\theta})=1$. We can obtain the maximum product of spacings estimators of the unknown parameters by maximizing the following function with respect to $\delta, \alpha$ and $\lambda$

$$
\begin{aligned}
M(\boldsymbol{\theta})= & \frac{1}{n+1} \sum_{i=1}^{n+1} \log \left[D_{i}(\boldsymbol{\theta})\right] \\
= & \frac{1}{n+1} \sum_{i=1}^{n+1} \log \\
& \left\{\frac{\log \left[\Psi\left(x_{i-1} ; \boldsymbol{\theta}\right)\right]-\log \left[\Psi\left(x_{i} ; \boldsymbol{\theta}\right)\right]}{\log (\delta)}\right\},
\end{aligned}
$$

where $\Psi\left(x_{i: n} ; \boldsymbol{\theta}\right) \equiv \Psi\left(x_{i} ; \boldsymbol{\theta}\right)$ for simplicity of notation.

4.4 Methods of ordinary and weighted leastsquares

The ordinary least squares and the weighted least square estimators of the LTIW parameters can be obtained by minimizing the following function

$L S(\boldsymbol{\theta})=\sum_{i=1}^{n} \omega_{i}\left\{\epsilon(i, n)-\frac{\log \left[\Psi\left(x_{i} ; \boldsymbol{\theta}\right)\right]}{\log (\delta)}\right\}^{2}$,

where $\epsilon(i, n)=\frac{n+1-i}{n+1}$ and $\omega_{i}=1$ in the case of ordinary least square method and $\omega_{i}=$ $\frac{(n+1)^{2}(n+2)}{i(n-i+1)}$ in the case of weighted least squares method.

\subsection{Methods of minimum distances}

In this subsection, we consider three methods of estimation for $\delta, \alpha$ and $\lambda$ by minimizing of the goodness-of-fit statistics with respect to $\delta, \alpha$ and $\lambda$.

\subsubsection{Method of Cramér-von-Mises}

The Cramér-von Mises estimates of the LTIW parameters are obtained by minimizing the following function:

$C(\boldsymbol{\theta})=\frac{1}{12 n}+\sum_{i=1}^{n}\left\{\varepsilon_{i}-\frac{\log \left[\Psi\left(x_{i} ; \boldsymbol{\theta}\right)\right]}{\log (\delta)}\right\}^{2}$,

with respect to $\delta, \alpha$ and $\lambda$, where $\varepsilon_{i}=\frac{n-i+0.5}{n}$. 
4.5.2 Methods of Anderson-Darling and righttail Anderson-Darling

The Anderson-Darling estimators, see Anderson \& Darling, 1952, of the unknown parameters of the LTIW distribution are obtained by minimizing the following function with respect to $\delta, \alpha$ and $\lambda$

$$
\begin{aligned}
A(\boldsymbol{\theta}) & =-n-\frac{1}{n} \sum_{i=1}^{n} \tau(i)\left\{\log F\left(x_{i: n} \mid \boldsymbol{\theta}\right)\right. \\
& \left.+\log \bar{F}\left(x_{n+1-i: n} \mid \boldsymbol{\theta}\right)\right\}
\end{aligned}
$$

where $\tau(i)=2 i-1$.

The Right-tail Anderson-Darling estimators of the LTIW distribution are obtained by minimizing the following function with respect to $\delta, \alpha$ and $\lambda$ :

$$
\begin{aligned}
R(\boldsymbol{\theta}) & =\frac{n}{2}-2 \sum_{i=1}^{n} F\left(x_{i: n} \mid \boldsymbol{\theta}\right) \\
& -\frac{1}{n} \sum_{i=1}^{n} \tau(i) \log \bar{F}\left(x_{n+1-i: n} \mid \boldsymbol{\theta}\right) .
\end{aligned}
$$

\subsection{Bayesian estimation}

In this subsection, we obtain the Bayes estimators of the unknown parameters of the LTIW distribution based on squared error loss function (SELF). The joint prior of $\delta, \alpha$ and $\lambda$ can be written as follows

$$
g(\delta, \alpha, \lambda) \propto \frac{1}{\delta \alpha \lambda}, \delta, \alpha, \lambda>0 .
$$

Based on the likelihood function in (11) and the joint prior in (16), we can write the joint posterior distribution as

$$
\begin{aligned}
g(\delta, \alpha, \lambda \mid \underline{x}) & =c^{-1} \lambda^{n-1} \alpha^{n-1} \delta^{\sum_{i=1}^{n} e^{-\lambda x_{i}^{-\alpha}}-1} \\
& \times e^{-(\alpha+1) \sum_{i=1}^{n} \log \left(x_{i}\right)-\lambda \sum_{i=1}^{n} x_{i}^{-\alpha}} \\
& \times \prod_{i=1}^{n}\left(\frac{1}{1+\delta-\delta^{-\lambda x_{i}^{-\alpha}}}\right),(17)
\end{aligned}
$$

where

$$
\begin{aligned}
c= & \int_{0}^{\infty} \int_{0}^{\infty} \int_{0}^{\infty} \lambda^{n-1} \alpha^{n-1} \delta^{\sum_{i=1}^{n} e^{-\lambda x_{i}^{-\alpha}}-1} \\
& e^{-(\alpha+1) \sum_{i=1}^{n} \log \left(x_{i}\right)-\lambda \sum_{i=1}^{n} x_{i}^{-\alpha}} \\
\times & \prod_{i=1}^{n}\left(\frac{1}{1+\delta-\delta^{e^{-\lambda x_{i}^{-\alpha}}}}\right) d \delta d \alpha d \lambda .
\end{aligned}
$$

Under SELF, we can obtain the Bayes estimators of the function $U(\delta, \alpha, \lambda)$ by

$$
\widehat{U}^{B S}(\delta, \alpha, \lambda)=E_{\delta, \alpha, \lambda \mid \underline{x}}[U(\delta, \alpha, \lambda)] .(18
$$

The MCMC technique is adopted to generate samples from the posterior distribution and in turn to obtain the Bayes estimates under squared error loss function.

$$
\begin{aligned}
g_{1}(\delta \mid \alpha, \lambda, \underline{x}) & \propto \delta^{\sum_{i=1}^{n} e^{-\lambda x_{i}^{-\alpha}}-1} \\
& \times \prod_{i=1}^{n}\left(\frac{1}{1+\delta-\delta^{e^{-\lambda x_{i}^{-\alpha}}}}\right), \\
g_{2}(\alpha \mid \delta, \lambda, \underline{x}) & \propto \alpha^{n-1} \delta^{\sum_{i=1}^{n} e^{-\lambda x_{i}^{-\alpha}}} \\
& \times e^{-(\alpha+1) \sum_{i=1}^{n} \log \left(x_{i}\right)-\lambda \sum_{i=1}^{n} x_{i}^{-\alpha}} \\
& \times \prod_{i=1}^{n}\left(\frac{1}{1+\delta-\delta^{-\lambda x_{i}^{-\alpha}}}\right)
\end{aligned}
$$

and

$$
\begin{aligned}
g_{3}(\lambda \mid \delta, \alpha, \underline{x}) & \propto \lambda^{n-1} \delta^{\sum_{i=1}^{n} e^{-\lambda x_{i}^{-\alpha}}} e^{-\lambda \sum_{i=1}^{n} x_{i}^{-\alpha}} \\
& \times \prod_{i=1}^{n}\left(\frac{1}{1+\delta-\delta^{e^{-\lambda x_{i}^{-\alpha}}}}\right) .
\end{aligned}
$$

It is noted that the above distributions are not known but Figure 3 shows that their graphs are similar to normal distribution. Therefore, we use the Metropolis-Hastings steps to generate random samples from these distributions as follows

1. Set $t=1$, and use the MLEs as initial values for $\delta, \alpha$ and $\lambda$

2. Use Metropolis Hastings algorithm to generate $\delta^{(t)}$ from $g_{1}(\delta \mid \alpha, \lambda, \underline{x})$ 
with normal proposal distribution as $N\left(\widehat{\delta}^{M L E}, \operatorname{var}\left(\widehat{\delta}^{M L E}\right)\right)$.

3. Use Metropolis Hastings algorithm to generate $\alpha^{(t)}$ from $g_{2}(\alpha \mid \delta, \lambda, \underline{x})$ with proposal distribution as $N\left(\widehat{\alpha}^{M L E}, \operatorname{var}\left(\widehat{\alpha}^{M L E}\right)\right)$.

4. Use Metropolis Hastings algorithm to generate $\lambda^{(t)}$ from $g_{3}(\lambda \mid \delta, \alpha, \underline{x})$ with proposal distribution as $N\left(\widehat{\lambda}^{M L E}, \operatorname{var}\left(\widehat{\lambda}^{M L E}\right)\right)$.

5. Set $t=t+1$.

6. Repeat step 2-5 $M$ times and obtain $\left(\delta^{(t)}, \alpha^{(t)}, \lambda^{(t)}\right), t=1,2, \ldots, M$.

7. Compute the Bayes estimates of $\delta, \alpha$ and $\lambda$ under SELF, denoted by $\widehat{\delta}^{B S}, \widehat{\alpha}^{B S}$ and $\widehat{\lambda}^{B S}$, as

$$
\begin{aligned}
\widehat{\delta}^{B S} & =\frac{1}{M-B} \sum_{t=B+1}^{M} \delta^{(k)}, \\
\widehat{\alpha}^{B S} & =\frac{1}{M-B} \sum_{t=B+1}^{M} \alpha^{(t)}, \\
\widehat{\lambda}^{B S} & =\frac{1}{M-B} \sum_{t=B+1}^{M} \lambda^{(t)},
\end{aligned}
$$

where $B$ is the burn-in period.

\section{Simulation results}

In this section, a simulation study is conducted to compare the performance of the different estimators in terms of their mean square errors (MSEs). The simulation study is conducted by considering different parameter values and different sample sizes. We choose the scale parameter $\lambda$ to be one in all the cases and $(\delta, \alpha)=(2,2),(0.5,1.5)$ and $(1.5,1.5)$.The sample sizes are considered to be $n=20,50,100,150,200$ and 200. The different estimates are obtained based on 1000 repetitions. The Bayes estimates are obtained using the MCMC technique based on 12000 samples with 2000 as a burn-in period. The average values of estimates and MSEs of the different methods are
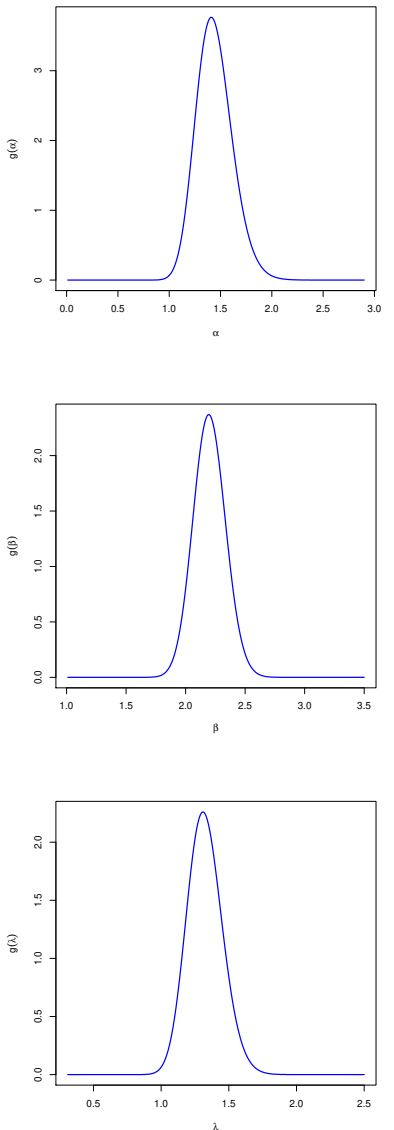

Fig. 3. Conditional posterior distributions of $\delta, \alpha$ and $\lambda$.

obtained and displayed in Tables 1-3.

The results in Tables 1-3 reveal interesting information. As the sample size increases the estimates are very close to the true parameters which indicates the asymptotically unbiased property of the different estimates except the percentile estimates. Also, the MSEs decrease in all the cases as the sample size increases which indicates that consistency of the different estimates. Comparing the different estimates we can conclude that the Bayes estimates have the minimum MSEs among the other estimates in most of the cases. It is also observed that the percentile estimates have the largest MSEs among the other estimates especially for the parameters $\delta$ and $\alpha$. 
Table 1. Average values of estimates (first row) along with the corresponding MSEs (second row) for $\delta=2, \alpha=2$ and $\lambda=1$.

\begin{tabular}{|c|c|c|c|c|c|}
\hline & \multirow{3}{*}{$\frac{\mathrm{Par}}{\delta}$} & Irs MLE PCE & \multicolumn{3}{|c|}{ WLSE CME ADE RADE BSE } \\
\hline & & 2.1261 .0662 .0262 .057 & & & 2.061 \\
\hline & & $\begin{array}{llll}0.718 & 0.972 & 0.766 & 0.768\end{array}$ & 0.7990 .7200 .749 & 0.983 & 0.705 \\
\hline & & $\begin{array}{llll}2.151 & 2.963 & 1.955 & 1.979\end{array}$ & $2.203 \quad 2.1072 .040$ & 2.574 & 2.298 \\
\hline & & $\begin{array}{llll}0.194 & 0.971 & 0.204 & 0.188\end{array}$ & 0.2040 .2230 .179 & 0.977 & 0.190 \\
\hline & \multirow[t]{2}{*}{$\lambda$} & $\begin{array}{llll}1.107 & 1.145 & 1.090 & 1.089\end{array}$ & $0.869 \quad 1.1311 .089$ & 0.709 & 1.138 \\
\hline & & $\begin{array}{llll}0.119 & 0.035 & 0.109 & 0.119\end{array}$ & 0.1200 .1180 .114 & 0.247 & 0.116 \\
\hline \multirow[t]{6}{*}{50} & \multirow[t]{2}{*}{$\delta$} & $2.048 \quad 1.136 \quad 1.9962 .004$ & $\begin{array}{lll}2.368 & 2.029 & 2.007\end{array}$ & 1.453 & 2.027 \\
\hline & & $\begin{array}{llll}0.577 & 0.791 & 0.599 & 0.600\end{array}$ & $0.661 \quad 0.5920 .579$ & 0.797 & 0.574 \\
\hline & \multirow[t]{2}{*}{$\alpha$} & $\begin{array}{llll}2.037 & 2.889 & 1.968 & 1.978\end{array}$ & 2.0752 .0341 .991 & 2.542 & 2.082 \\
\hline & & $\begin{array}{llll}0.075 & 0.800 & 0.100 & 0.088\end{array}$ & 0.0740 .1 & 0.79 & 0.074 \\
\hline & \multirow[t]{2}{*}{$\lambda$} & $\begin{array}{llll}1.083 & 1.126 & 1.080 & 1.082\end{array}$ & 0.9251 .0931 .083 & 0.750 & 1.142 \\
\hline & & $\begin{array}{llll}0.075 & 0.023 & 0.069 & 0.073\end{array}$ & $0.070 \quad 0.070 \quad 0.071$ & 0.157 & 0.071 \\
\hline \multirow[t]{6}{*}{100} & \multirow[t]{2}{*}{$\delta$} & $2.073 \quad 1.211 \quad 2.064 \quad 2.051$ & $\begin{array}{lll}2.325 & 2.083 & 2.056\end{array}$ & 1.590 & 1.937 \\
\hline & & $\begin{array}{llll}0.445 & 0.628 & 0.438 & 0.444\end{array}$ & $0.468 \quad 0.436$ & 0.62 & 0.443 \\
\hline & \multirow[t]{2}{*}{$\alpha$} & 2.0192 .7901 .98 & 2.0462. & 2.4 & 1.936 \\
\hline & & $\begin{array}{llll}0.038 & 0.637 & 0.051 & 0.042\end{array}$ & 0.0360 .0530 .041 & 0.630 & 0.037 \\
\hline & \multirow[t]{2}{*}{$\lambda$} & $\begin{array}{llll}1.032 & 1.079 & 1.026 & 1.033\end{array}$ & 0.9201 .0321 .032 & 0.847 & 0.932 \\
\hline & & $\begin{array}{llll}0.046 & 0.010 & 0.045 & 0.046\end{array}$ & 0.0450 .0440 .044 & 0.088 & 0.045 \\
\hline \multirow[t]{6}{*}{150} & \multirow[t]{2}{*}{$\delta$} & $2.031 \quad 1.323 \quad 1.9862 .030$ & 2.2012 .0022 .007 & 1.741 & 2.045 \\
\hline & & 0.28 & 0.3 & & 0.282 \\
\hline & \multirow[t]{2}{*}{$\alpha$} & $\begin{array}{llll}33 & 1.983 & 1.992\end{array}$ & 2.0312 .0061 & 2.25 & 2.064 \\
\hline & & $\begin{array}{llll}0.025 & 0.486 & 0.034 & 0.029\end{array}$ & 0.0240 .0340 .028 & 0.483 & 0.025 \\
\hline & \multirow[t]{2}{*}{$\lambda$} & $\begin{array}{lllll}1.019 & 1.047 & 1.027 & 1.024\end{array}$ & $0.945 \quad 1.0301 .024$ & 0.927 & 1.020 \\
\hline & & $\begin{array}{llll}0.025 & 0.005 & 0.024 & 0.024\end{array}$ & 0.0250 .0240 .024 & 0.039 & 0.024 \\
\hline \multirow[t]{6}{*}{200} & \multirow[t]{2}{*}{$\delta$} & $\begin{array}{llll}2.066 & 1.359 & 2.059 & 2.062\end{array}$ & 2.1762 .0702 .060 & 1.76 & 2.014 \\
\hline & & & 0.215 & 0.3 & 0.201 \\
\hline & \multirow[t]{2}{*}{$\alpha$} & $2.0112 .599 \quad 1.99$ & 2.0192 .0122 .0 & 2.183 & 2.001 \\
\hline & & $\begin{array}{lllll}0.020 & 0.359 & 0.027 & 0.023\end{array}$ & $\begin{array}{llll}0.020 & 0.028 & 0.022\end{array}$ & 0.377 & 0.021 \\
\hline & \multirow[t]{2}{*}{$\lambda$} & $\begin{array}{llll}1.009 & 1.018 & 1.008 & 1.008\end{array}$ & 0.9581 .0111 .009 & 0.951 & 0.990 \\
\hline & & 0.015 & 0.015 & 0.02 & 0.014 \\
\hline \multirow[t]{6}{*}{250} & $\delta$ & $\begin{array}{lllll}2.038 & 1.462 & 2.027 & 2.029\end{array}$ & $\begin{array}{lll}2.100 & 2.039 & 2.032\end{array}$ & 1.85 & 2.045 \\
\hline & & $\begin{array}{llll}0.120 & 0.298 & 0.122 & 0.119\end{array}$ & 0.1180 .1220 .11 & 0.27 & 0.119 \\
\hline & $\alpha$ & $2.0042 .498 \quad 1.986 \quad 1.991$ & 2.0062 .0001 .9 & 2.10 & 2.003 \\
\hline & & $\begin{array}{llll}0.013 & 0.249 & 0.017 & 0.0\end{array}$ & $0.0120 .017 \quad 0.015$ & 0.267 & 0.011 \\
\hline & $\lambda$ & 1.0021 .003 & $0.971 \quad 1.0$ & 0.97 & 0.993 \\
\hline & & 0.0080 .0020 .00 & 0.0080 .0070 .007 & 0.01 & 0.001 \\
\hline
\end{tabular}

\section{Real data applications}

In this section, we prove the flexibility of the LTIW distribution in modelling real data and compare it with some other competitive models, which are given in Table 4. The following five measures are considered for model comparison including $-\hat{\ell}$ (where $\ell$ is the maximized log-likelihood), $W^{*}$ (Cramér-von Mises), $A^{*}$ (Anderson-Darling), AIC (Akaike information criterion), and $B I C$ (Bayesian information criterion).

The first set of data was studied by Feigl and Zelen, 1965, and it refers to survival times (in weeks) of 33 patients suffering from acute Myelogeneous leukaemia.

The second set of data consists of 40 observations, and refers to the October maximum flows (m3/s) on the Piracicaba river, which cover
Table 2. Average values of estimates (first row) along with the corresponding MSEs (second row) for $\delta=0.5, \alpha=1.5$ and $\lambda=1$.

$n$ Pars MLE PCE MPSE LSE WLSE CME ADE RADE BSE

\begin{tabular}{lllllllllll}
\hline 20 & $\delta$ & 0.570 & 0.150 & 0.503 & 0.487 & 0.681 & 0.491 & 0.506 & 0.101 & 0.477
\end{tabular} $\begin{array}{lllllllllll}0.083 & 0.151 & 0.083 & 0.086 & 0.104 & 0.080 & 0.086 & 0.159 & 0.083\end{array}$

$\begin{array}{llllllllll}\alpha & 1.597 & 1.868 & 1.411 & 1.414 & 1.650 & 1.506 & 1.463 & 1.130 & 1.621\end{array}$ $\begin{array}{lllllllll}0.077 & 0.154 & 0.080 & 0.078 & 0.076 & 0.073 & 0.074 & 0.149 & 0.074\end{array}$

$\begin{array}{llllllllll}\lambda & 1.006 & 1.295 & 1.117 & 1.147 & 0.807 & 1.119 & 1.119 & 0.502 & 1.025\end{array}$ $\begin{array}{llllllllll}0.111 & 0.118 & 0.118 & 0.135 & 0.120 & 0.117 & 0.134 & 0.248 & 0.109\end{array}$

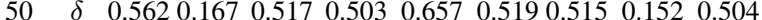
$\begin{array}{llllllllll}0.066 & 0.119 & 0.064 & 0.067 & 0.077 & 0.064 & 0.067 & 0.122 & 0.062\end{array}$

$\begin{array}{llllllllll}\alpha & 1.546 & 1.835 & 1.455 & 1.456 & 1.592 & 1.502 & 1.475 & 1.215 & 1.633\end{array}$ $\begin{array}{lllllllll}0.048 & 0.120 & 0.048 & 0.049 & 0.047 & 0.049 & 0.048 & 0.083 & 0.042\end{array}$

$\begin{array}{lllllllllll}\lambda & 1.015 & 1.203 & 1.079 & 1.104 & 0.871 & 1.071 & 1.087 & 0.604 & 0.926\end{array}$ $\begin{array}{llllllllll}0.096 & 0.065 & 0.095 & 0.110 & 0.091 & 0.095 & 0.107 & 0.159 & 0.087\end{array}$

\begin{tabular}{lllllllllll}
\hline 100 & $\delta$ & 0.560 & 0.207 & 0.517 & 0.522 & 0.626 & 0.521 & 0.527 & 0.202 & 0.519
\end{tabular} $\begin{array}{llllllllll}0.053 & 0.089 & 0.050 & 0.053 & 0.059 & 0.050 & 0.052 & 0.089 & 0.053\end{array}$

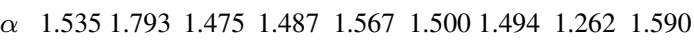
$\begin{array}{llllllllll}0.035 & 0.089 & 0.035 & 0.035 & 0.034 & 0.035 & 0.034 & 0.057 & 0.032\end{array}$

$\begin{array}{lllllllllll}\lambda & 1.001 & 1.066 & 1.058 & 1.055 & 0.906 & 1.050 & 1.046 & 0.653 & 0.968\end{array}$ $\begin{array}{lllllllllll}0.076 & 0.023 & 0.074 & 0.081 & 0.072 & 0.074 & 0.079 & 0.121 & 0.066\end{array}$

\begin{tabular}{lllllllllll}
\hline 150 & $\delta$ & 0.538 & 0.253 & 0.506 & 0.509 & 0.588 & 0.511 & 0.511 & 0.264 & 0.537
\end{tabular} $\begin{array}{lllllllll}0.040 & 0.062 & 0.036 & 0.039 & 0.041 & 0.036 & 0.039 & 0.062 & 0.037\end{array}$

$\begin{array}{llllllllll}\alpha & 1.525 & 1.747 & 1.483 & 1.490 & 1.550 & 1.501 & 1.492 & 1.313 & 1.536\end{array}$ $\begin{array}{lllllllll}0.024 & 0.062 & 0.024 & 0.024 & 0.024 & 0.024 & 0.024 & 0.036 & 0.025\end{array}$

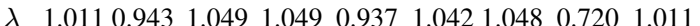
$\begin{array}{llllllllll}0.057 & 0.018 & 0.054 & 0.057 & 0.050 & 0.052 & 0.058 & 0.091 & 0.053\end{array}$

\begin{tabular}{llllllllllll}
\hline 200 & $\delta$ & 0.537 & 0.301 & 0.508 & 0.511 & 0.576 & 0.508 & 0.515 & 0.352 & 0.535
\end{tabular} $\begin{array}{llllllllll}0.024 & 0.040 & 0.023 & 0.024 & 0.025 & 0.023 & 0.024 & 0.040 & 0.021\end{array}$

$\begin{array}{llllllllll}\alpha & 1.530 & 1.698 & 1.493 & 1.502 & 1.549 & 1.504 & 1.505 & 1.370 & 1.523\end{array}$ $\begin{array}{llllllllll}0.015 & 0.040 & 0.016 & 0.016 & 0.016 & 0.016 & 0.016 & 0.020 & 0.011\end{array}$

$\begin{array}{llllllllll}\lambda & 0.988 & 0.836 & 1.026 & 1.022 & 0.933 & 1.024 & 1.017 & 0.828 & 0.986\end{array}$ $\begin{array}{lllllllllll}0.035 & 0.039 & 0.034 & 0.035 & 0.034 & 0.034 & 0.035 & 0.070 & 0.031\end{array}$

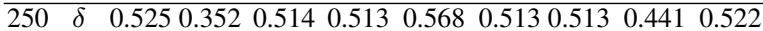
$\begin{array}{lllllllll}0.015 & 0.022 & 0.014 & 0.014 & 0.023 & 0.014 & 0.014 & 0.022 & 0.011\end{array}$

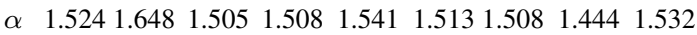
$\begin{array}{lllllllllll}0.009 & 0.022 & 0.008 & 0.008 & 0.015 & 0.009 & 0.008 & 0.010 & 0.008\end{array}$

$\begin{array}{lllllllllll}\lambda & 0.989 & 0.818 & 1.004 & 1.006 & 0.948 & 1.005 & 1.006 & 0.952 & 0.987\end{array}$ $\begin{array}{llllllllll}0.019 & 0.037 & 0.018 & 0.019 & 0.034 & 0.018 & 0.018 & 0.055 & 0.011\end{array}$

the period from 1960 to 2014 .

The numerical values of $-\hat{\ell}, W^{*}, A^{*}, A I C$ and $B I C$, the MLEs and their associated standard errors (SEs) (in parentheses) of all fitted distributions are shown in Tables 5 and 6, for the given data sets respectively.

The fitted PDF and the estimated survival function of the LTIW distribution are displayed in Figure 4 for the two data sets, respectively. The results in Tables 5 and 6 show that the LTIW distribution provides a close fits to the two data sets.

\section{Concluding remarks}

In this paper, we propose a three-parameter model, called the logarithmic transformed inverse Weibull (LTIW) distribution. The proposed LTIW distribution has two shape parameters and one scale parameter. The 
Table 3. Average values of estimates (first row) along with the corresponding MSEs (second row) for $\delta=1.5, \alpha=0.5$ and $\lambda=1$.

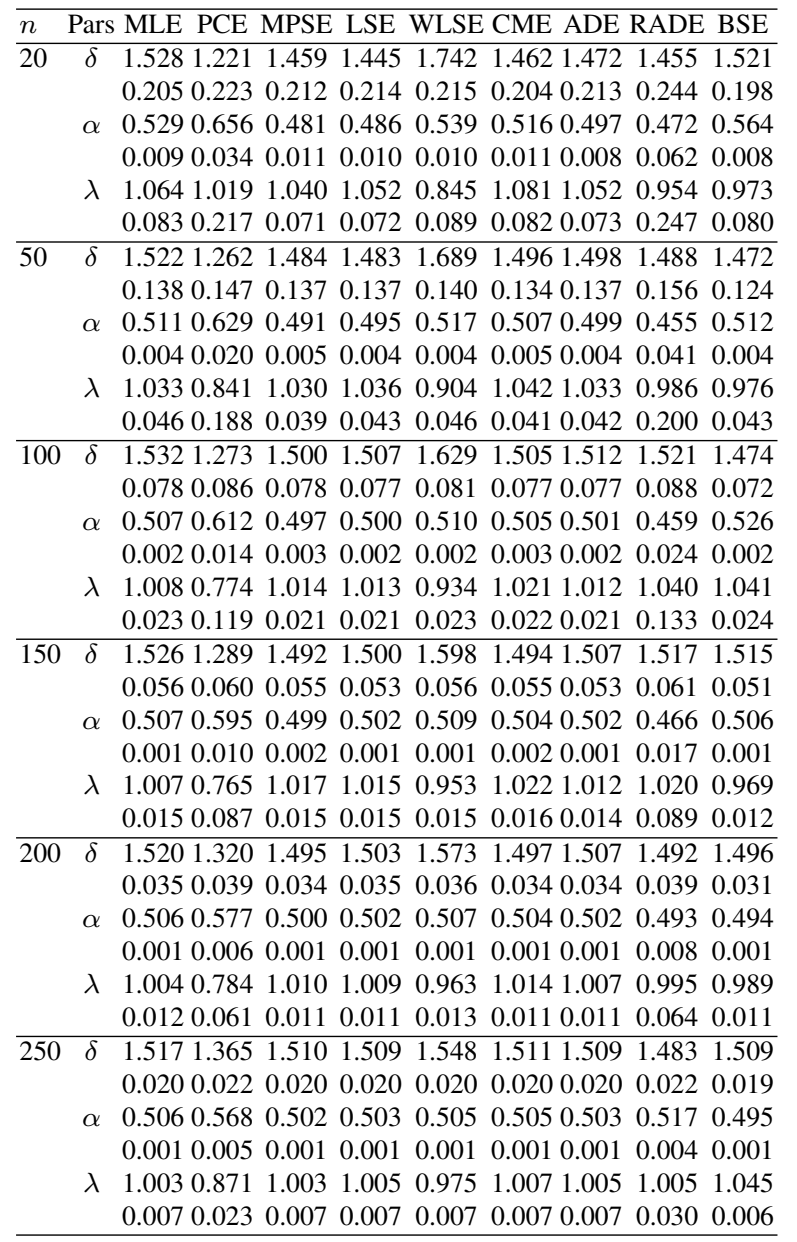

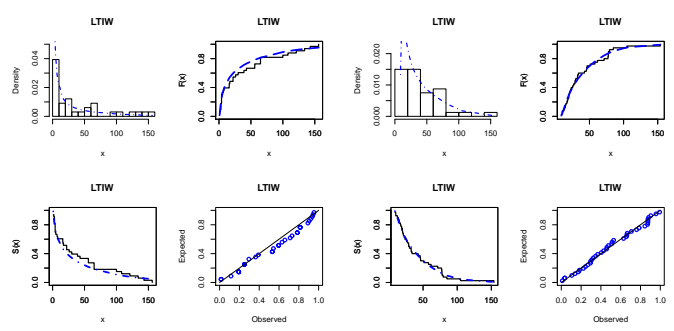

Fig. 4. Fitted density, estimated CDF and SF, and P-P plots (a) for leukaemia data and (b) for Piracicaba river data.

LTIW distribution includes some special submodels namely, the inverse Weibull, inverse exponential, inverse Rayleigh, logarithmic transformed inverse exponential and logarithmic transformed inverse Rayleigh distributions. The LTIW density function can be expressed as a mixture of IW densities. The model parameters are estimated by nine estimation methods
Table 4. Some competitive models of the LTIW distribution.

\begin{tabular}{lcl}
\hline \hline Distribution & Abbreviation Author(s) \\
\hline Burr X Fréchet & BXFr & Abouelmagd et al., (2018) \\
Weibull Fréchet & WFr & Afify et al., (2016) \\
Modified Fréchet & TGW & Tablada \& Cordeiro (2017) \\
Odd Lindley Fréchet & OLFr & Mansour et al., (2018) \\
Exponentiated Fréchet & EFr & Nadarajah \& Kotz (2003) \\
Marshall-Olkin Fréchet & MOFr & Krishna et al., (2013) \\
Generalized inverse Weibull & GIW & De Gusmao et al., (2011) \\
Inverse Weibull & IW & Fréchet (1924) \\
\hline \hline
\end{tabular}

Table 5. Goodness-of-fit statistics, MLEs and (SEs) for leukaemia data.

\begin{tabular}{|c|c|c|c|c|c|c|}
\hline Model & $-\hat{\ell}$ & $W^{*}$ & $A^{*}$ & $A I C$ & $B I C$ & Estimates (SEs) \\
\hline \multirow[t]{3}{*}{ LTIW } & 150.752 & 0.0514 & 0.3917 & 307.504 & 309.015 & $\delta 6442500(114.8)$ \\
\hline & & & & & & $\alpha 3.1891(0.2073)$ \\
\hline & & & & & & $\lambda 0.1061(0.0683)$ \\
\hline \multirow[t]{3}{*}{ BXFr } & 151.079 & 0.0654 & 0.4267 & 308.157 & 312.647 & $\alpha 62.261(3.2345)$ \\
\hline & & & & & & $\beta 0.5805(0.0852)$ \\
\hline & & & & & & $\theta 0.1715(0.0511)$ \\
\hline \multirow[t]{4}{*}{ WFr } & 151.289 & 0.0613 & 0.4255 & 310.577 & 316.564 & $\alpha 2.6221(1.8334)$ \\
\hline & & & & & & $\beta 1.8388(1.4643)$ \\
\hline & & & & & & $a 0.1703(0.1457)$ \\
\hline & & & & & & $b 0.3807(0.2711)$ \\
\hline \multirow[t]{3}{*}{ MFr } & 151.562 & 0.0711 & 0.4705 & 309.124 & 313.613 & $\alpha 14.231(7.4633)$ \\
\hline & & & & & & $\beta 0.4634(0.1138)$ \\
\hline & & & & & & $\theta 0.0123(0.0050)$ \\
\hline \multirow[t]{3}{*}{ OLFr } & 152.644 & 0.0870 & 0.5694 & 311.288 & 315.777 & $\alpha 9.2691(12.600)$ \\
\hline & & & & & & $\beta 0.4952(0.1025)$ \\
\hline & & & & & & $\theta$ 1.1197(0.9916) \\
\hline \multirow[t]{3}{*}{$\mathrm{EFr}$} & 153.894 & 0.1115 & 0.7051 & 313.788 & 318.277 & $\alpha 1426.6(3605.6)$ \\
\hline & & & & & & $\beta 0.2491(0.0707)$ \\
\hline & & & & & & $\lambda 13.746(13.505)$ \\
\hline \multirow[t]{3}{*}{ MOFr } & 154.689 & 0.1289 & 0.7978 & 315.378 & 319.868 & $\alpha 1.9073(1.8015)$ \\
\hline & & & & & & $\beta 0.9875(0.1859)$ \\
\hline & & & & & & $\theta 8.0680(11.147)$ \\
\hline \multirow[t]{3}{*}{ GIW } & 155.999 & 0.1601 & 0.9759 & 317.997 & 322.487 & $\alpha 2.2379(487.94)$ \\
\hline & & & & & & $\beta 0.6944(0.0914)$ \\
\hline & & & & & & $\lambda 2.4656(774.18)$ \\
\hline \multirow[t]{2}{*}{ IW } & 155.999 & 0.1601 & 0.9759 & 315.997 & 318.990 & $\beta 0.6944(0.0914)$ \\
\hline & & & & & & $\lambda 4.1878(0.9027)$ \\
\hline
\end{tabular}

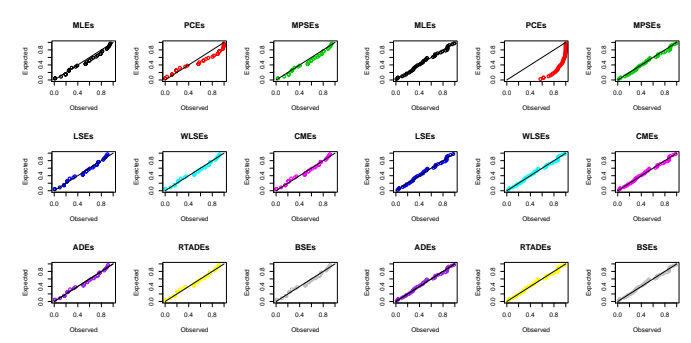

Fig. 5. P-P plots of the LTIW distribution using various methods (a) for leukaemia data and (b) for Piracicaba river data.

including the Bayesian method. To compare the performance of the different estimators a simulation study is conducted. The simulation 
Table 6. Goodness-of-fit statistics, MLEs and (SEs) for Piracicaba river data.

\begin{tabular}{|c|c|c|c|c|}
\hline Model & $-\widehat{\ell} \quad W^{*}$ & $A^{*}$ & $A I C \quad B I C$ & Estimates (SEs) \\
\hline \multirow[t]{3}{*}{ LTIW } & 180.2370 .0334 & 0.2382 & 366.474371 .540 & $\delta 366659.7(561.6)$ \\
\hline & & & & $\alpha 5.6064(0.1411)$ \\
\hline & & & & $\lambda$ 33702.4(106.08) \\
\hline \multirow[t]{3}{*}{ BXFr } & 181.9860 .0504 & 0.3446 & 369.973375 .039 & $\alpha 0.1307(0.5648)$ \\
\hline & & & & $\beta 0.1653(0.0863)$ \\
\hline & & & & $\theta$ 38.053(89.721) \\
\hline \multirow[t]{4}{*}{ WFr } & 180.5580 .0338 & 0.2402 & 369.116375 .872 & $\alpha$ 19.789(7.8930) \\
\hline & & & & $\beta 2.4388(1.4465)$ \\
\hline & & & & $a 0.4283(0.3579)$ \\
\hline & & & & $b 0.4798(0.2223)$ \\
\hline \multirow[t]{3}{*}{ MFr } & 181.4220 .0427 & 0.2954 & 368.844373 .911 & $\alpha 35.947(16.330)$ \\
\hline & & & & $\beta 1.0496(0.3501)$ \\
\hline & & & & $\theta 0.0168(0.0106)$ \\
\hline \multirow[t]{3}{*}{ OLFr } & 181.7320 .0604 & 0.3765 & 369.464374 .531 & $\alpha 50.618(49.031)$ \\
\hline & & & & $\beta 0.8139(0.2641)$ \\
\hline & & & & $\theta 2.8089(3.1122)$ \\
\hline \multirow[t]{3}{*}{$\mathrm{EFr}$} & 182.0690 .0524 & 0.3558 & 370.138375 .205 & $\alpha 86.109(149.80)$ \\
\hline & & & & $\beta 0.7294(0.4696)$ \\
\hline & & & & $\lambda 5.2605(8.7727)$ \\
\hline \multirow[t]{3}{*}{ MOFr } & 182.2220 .0482 & 0.3534 & 370.443375 .510 & $\alpha$ 14.114(5.2417) \\
\hline & & & & $\beta 2.0396(0.3998)$ \\
\hline & & & & $\theta 4.3924(5.2489)$ \\
\hline \multirow[t]{3}{*}{ GIW } & 182.9600 .0527 & 0.4212 & 371.920376 .978 & $\alpha 6.8532(4140.3)$ \\
\hline & & & & $\beta 1.5619(0.1888)$ \\
\hline & & & & $\lambda 6.6263(2563.0)$ \\
\hline \multirow[t]{2}{*}{ IW } & 182.9600 .0527 & 0.4212 & 369.920373 .298 & $\beta 1.5616(0.1887)$ \\
\hline & & & & $\lambda$ 131.29(73.204) \\
\hline
\end{tabular}

showed that the Bayes estimates perform better than other classical estimates in terms of minimum mean square errors. Finally, two real data sets are analyzed to show the applicability of the proposed model and the different estimators discussed.

\section{References}

Abouelmagd, T.H.M.; Hamed, M.S.; Afify, A.Z.; Al-Mofleh, H. \& Iqbal, Z. (2018). The Burr X Fréchet distribution with its properties and applications. Journal of Applied Probability, 13: 23-51.

Afify, A.Z.; Yousof, H.M.; Cordeiro, G.M.; Ortega, E.M.M. \& Nofal, Z.M. (2016). The Weibull Fréchet distribution and its applications. Journal of Applied Statistics, 43: 2608-2626.

Anderson, T.W. \& Darling, D.A. (1952). Asymptotic theory of certain goodness of fit criteria based on stochastic processes. The annals of mathematical statistics, 23: 193-212.
Feigl, P. \& Zelen, M. (1965). Estimation of exponential probabilities with concomitant information. Biometrics, 21: 826-838.

Fréchet, M. (1924). Sur la Loi des Erreurs d'Observation. Bulletin de la Soci et e Math ematique de Moscou, 33: 5-8.

Johnson, N.L.; Kotz, S. \& Balakrishnan, N. (1995). Distributions. In Continuous Univariate Distributions, 2nd ed., Vol. 2, Wiley, New York, NY, USA.

Krishna, E.; Jose, K.K.; Alice, T. \& Ristic, M.M. (2013). The Marshall-Olkin Fréchet distribution. Commun. Stat. Theory Methods, 42: 4091-4107.

Mansour, M.M.; Elrazik, E.M.A.; Altun, E.; Afify, A.Z. \& Iqbal, Z. (2018). A new three-parameter Fréchet distribution: properties and applications. Pak. J. Stat., 34: 441-458.

Nadarajah, S. \& Kotz, S. (2003). The exponentiated Fréchet distribution. Interstat Electronic Journal, 14; 1-7.

Nassar, M.; Dey, S. \& Kumar, D. (2018). Logarithm transformed Lomax distribution with applications. Calcutta Statistical Association Bulletin, 70: 122-135.

Okasha, H.M.; El-Baz, A.H.; Tarabia, A.M.K. \& Basheer, A.M. (2017). Extended inverse Weibull distribution with reliability application. Journal of the Egyptian Mathematical Society, 25: 343-349.

Tablada, C.J. \& Cordeiro, G.M. (2017). The modified Fréchet distribution and its properties. Commun. Stat. Theory Methods, 46: 1061710639.

Submitted: $\quad 05 \backslash 06 \backslash 2020$

Revised: $\quad 26 \backslash 08 \backslash 2020$

Accepted: $\quad 27 \backslash 08 \backslash 2020$

DOI: $\quad 10.48129 /$ kjs.v48i3.9896 